\title{
IVIII Predictors of Pharmacotherapy for Tobacco Use Among Veterans Admitted for COPD: The Role of Disparities and Tobacco Control Processes
}

\author{
Anne C. Melzer, $M D^{1,2}$, Laura C. Feemster, MD, MS ${ }^{1,2}$, Margaret P. Collins, PhD', and David H. Au, \\ $M D, M S^{1,2}$ \\ 'Division of Pulmonary and Critical Care, University of Washington, Seattle, WA, USA; ${ }^{2}$ Center of Innovation for Veteran-Centered and Value-Driven \\ Care, VA Puget Sound Health Care System, Seattle, WA, USA.
}

BACKGROUND: Many smokers admitted for chronic obstructive pulmonary disease (COPD) are not given smoking cessation medications at discharge. The reasons behind this are unclear, and may reflect an interplay of patient characteristics, health disparities, and the receipt of inpatient tobacco control processes.

OBJECTIVES: We aimed to assess potential disparities in treatment for tobacco use following discharge for COPD, examined in the context of inpatient tobacco control processes.

PARTICIPANTS: Smokers aged $\geq 40$ years, admitted for treatment of a COPD exacerbation within the VA Veterans Integrated Service Network 20, identified using ICD-9 discharge codes and admission diagnoses from 2005-2012. MAIN MEASURES: The outcome was any tobacco cessation medication dispensed within 48 hours of discharge. We assessed potential predictors administratively up to 1 year prior to admission. We created the final logistic regression model using manual model building, clustered by site. Variables with $p<0.2$ in biviariate models were considered for inclusion in the final model.

RESULTS: We identified 1511 subjects. $16.9 \%$ were dispensed a medication at discharge. In the adjusted model, several predictors were associated with decreased odds of receiving medications: older age (OR per year older 0.96 , 95 \% CI 0.95-0.98), black race (OR 0.34, $95 \%$ CI 0.120.97), higher comorbidity score (OR 0.89, $95 \%$ CI 0.82 0.96), history of psychosis (OR 0.40, $95 \%$ CI 0.31-0.52), hypertension (OR 0.75, $95 \%$ CI 0.62-0.90), and treatment with steroids in the past year (OR 0.80, $95 \% \mathrm{CI}$ 0.70-0.90). Inpatient tobacco control processes were associated with increased odds of receiving medications: documented brief counseling at discharge (OR 3.08, 95 $\%$ CI 2.02-4.68) and receipt of smoking cessation medications while inpatient (OR 5.95, $95 \%$ CI 3.19-11.10).

CONCLUSIONS: Few patients were treated with tobacco cessation medications at discharge. We found evidence for disparities in treatment, but also potentially beneficial effects of inpatient tobacco control measures. Further

Electronic supplementary material The online version of this article (doi:10.1007/s11606-016-3623-4) contains supplementary material, which is available to authorized users.

Received July 22, 2015

Revised December 11, 2015

Accepted February 4, 2016

Published online February 22, 2016 focus should be on using novel processes of care to improve provision of medications and decrease the observed disparities.

KEY WORDS: COPD; tobacco; smoking; pharmacotherapy; nicotine replacement; disparities; processes of care.

J Gen Intern Med 31(6):623-9

DOI: $10.1007 / \mathrm{s} 11606-016-3623-4$

(c) Society of General Internal Medicine 2016

\section{BACKGROUND AND SIGNIFICANCE}

Chronic obstructive pulmonary disease (COPD) is now the third leading cause of death nationally. ${ }^{1}$ Tobacco cessation has been shown to be the most important intervention to slow the progression of disease. ${ }^{2}$ Unfortunately, up to one-third of patients admitted to the hospital for a COPD exacerbation continue to smoke. ${ }^{3,4}$ Hospitalization is a potentially effective time to provide evidence-based treatment for tobacco cessation. ${ }^{5}$ Patients are already in a tobacco-free environment with access to nurses, physicians and pharmacists able to facilitate treatment. However, the number of patients treated for tobacco cessation following hospitalization is very low. ${ }^{6}$

The reasons underlying the low rate of treatment for tobacco abuse among hospitalized patients are largely unknown, and likely reflect a complex interaction of patient and clinician behaviors, as well as the results of tobacco control efforts on the part of hospitals. Studies of the treatment of tobacco abuse in the outpatient setting have demonstrated significant disparities in the provision of medications for tobacco cessation by age, race, ${ }^{7}$ sex, presence of psychiatric disorders, ${ }^{8}$ tobaccorelated diagnoses, 9,10 and a number of other patient characteristics. COPD frequently coexists with other health disparities, ${ }^{11}$ and may itself be a risk factor for health-related disparities. Little data exists as to whether the disparities in treatment seen in the outpatient setting persist in the inpatient setting, and how they might interact with inpatient tobacco control efforts. While there are a number of studies examining the use of inpatient tobacco control measures, there is less data on the association of these measures with treatment after discharge. It is therefore important to examine the interplay of inpatient tobacco control measures in context with other patient 
characteristics that may influence treatment for tobacco addiction after discharge.

The goals of this study were to assess the existence of disparities in treatment with tobacco cessation medications following discharge for exacerbation of COPD, examining a range of patient characteristics, and to assess the association of inpatient tobacco control processes with post-discharge treatment for tobacco use in the context of these patient characteristics.

\section{METHODS}

\section{Design, Setting and Participants}

We conducted a cohort study of current smokers discharged from hospital for a COPD exacerbation within the Veterans Affairs (VA) Veterans Integrated Service Network (VISN)-20. This study was approved by the VA Puget Sound Health Care System Institutional Review Board (\#00461).

\section{Data Source}

We utilized information from the VISN-20 data warehouse that collects data using the VA electronic medical record, commonly utilized for research purposes. ${ }^{12-14}$ The warehouse obtained information on demographics, pharmacy records of medications dispensed, hospital and outpatient diagnoses, and dates of death. In addition, we utilized "health factors," electronic entries coded by staff at the time of an encounter to describe patient health behaviors, and the text of chart notes that were available for electronic query and review.

\section{Study Cohort}

We identified all patients aged $\geq 40$ hospitalized within the VISN-20 between 2005 and 2012 with a primary discharge diagnosis of COPD based on ICD-9 codes (491, 492, 493.2, and 496), or a diagnosis from the admission note indicating an exacerbation of COPD. Specific query terms for the admission diagnoses were selected based on consensus among several clinicians as appropriately indicating COPD exacerbation. We limited inclusion to current smokers aged $\geq 40$ years to improve the specificity of COPD diagnosis, excluding patients who died during the initial admission or had no smoking status identified.

To establish tobacco status, we built on previously developed and validated methodology ${ }^{15}$ and performed truncated natural language processing, identifying phrases in the medical record that reflected patients' tobacco status and querying all notes from the day of admission up to 6 months prior. We manually examined these records to confirm the tobacco status. If no status was indicated in the notes, we used the status encoded by the most recent health factor. This combined method allowed us to identify a baseline status for all but 77 of the 3580 patients admitted for COPD.

\section{Outcome and Exposures}

In order to assess prescriptions associated with the hospitalization, the primary outcome was the dispensation by the pharmacy of any approved medication to aid in smoking cessation within 48 hours of discharge, including: nicotine patch, short-acting nicotine replacement therapy (NRT), varenicline and buproprion.

Potential predictors were assessed administratively in the year prior to the index date or during the index hospitalization. We examined a range of individual characteristics and markers of tobacco control efforts, including: demographics (age, sex, race), psychosocial characteristics (history of drug or alcohol abuse, homelessness, depression, psychosis, or post-traumatic stress disorder [PTSD]), ${ }^{16}$ smoking-related comorbidities (Charlson comorbidity index, ${ }^{17}$ history of coronary artery disease, congestive heart failure, hypertension, stroke, or lung cancer), markers of COPD severity (prescription for steroids in the past year, mechanical or noninvasive ventilation during hospitalization, count of controller inhalers prior to admission), and inpatient tobacco control processes (treatment with smoking cessation medications as an inpatient, nurse-based counseling prior to discharge). Counseling at discharge was determined by nurses recording completion of a discharge process focused on smoking cessation. The presence of comorbid and psychiatric conditions were assessed using a validated method utilizing ICD-9 codes. ${ }^{16}$

\section{Statistical Analysis}

We used Stata 13 (College Station, TX) software. Chisquared tests and t-tests were used to assess bivariate associations. We built a logistic regression model using methods as described in Applied Logistic Regression Third Edition. ${ }^{18}$ In the initial model, we included all variables that attained $p \leq 0.2$ in bivariate models containing each predictor and the outcome of interest, clustered by site, with robust standard errors. We then created another preliminary model dropping all variables with $p>0.1$ in the initial multivariable model, retaining key demographic variables (age, sex, race), regardless of significance. We examined the $\beta$ coefficients to determine whether a $20 \%$ change was found when comparing this to the initial full model. We then re-introduced individual variables to the model to try to account for this variation in a parsimonious manner. Nested models were compared with robust Wald tests. Finally, each excluded variable was once again added back to the model to ensure that no significant variables had been excluded, yielding the final model. Model specification was assessed using the linktest function. The area under the curve of the final model was 0.77 , with $84.1 \%$ of subjects correctly categorized. The final regression equation is available in the Online Appendix 1. 


\section{Sensitivity Analyses}

Buproprion has dual indications for smoking cessation and as an antidepressant. Given that there may have been misclassification in the exposure and outcome related to the use of buproprion in this capacity, we performed sensitivity analyses excluding dispensation of buproprion at discharge or during the hospital stay. In addition, due to concerns that the inclusion of subjects with an admission diagnosis of COPD may have created a less specific cohort of subjects with COPD, we performed sensitivity analysis repeating the logistic regression model building exercise among only those subjects with a primary discharge diagnosis code of COPD.

\section{RESULTS}

We identified 1511 patients who met our inclusion criteria. Of these, $255(16.9 \%)$ patients were dispensed a tobacco cessation medication within 48 hours of discharge. Overall, patients were primarily older white males. In unadjusted analyses, patients who were dispensed a medication were on average younger and more likely to be white, with a lower mean Charlson index. Patients who were dispensed a medication were less likely to carry a diagnosis of stroke, congestive heart failure, hypertension, or coronary artery disease. Patients who were dispensed a medication at discharge were more likely to have been treated with tobacco cessation medications as an inpatient and to receive documented brief counseling from the nurse as part of the discharge process. (Table 1)

\section{Use of Medications}

Overall, $17.5 \%(n=264)$ of patients were treated with a tobacco cessation medication while inpatient. During the inpatient stay, the majority of those treated received the nicotine patch or buproprion, with minimal use of other tobacco

Table 1. Characteristics of Veterans Dispensed or Not Dispensed Medications for Tobacco Addiction Within 48 Hours of Discharge Following Exacerbation of COPD*

\begin{tabular}{|c|c|c|c|}
\hline \multirow[t]{2}{*}{ Variable } & \multicolumn{3}{|c|}{ Cessation medications dispensed at discharge } \\
\hline & $\begin{array}{l}\text { Yes } \\
n=255\end{array}$ & $\begin{array}{l}\text { No } \\
n=1256\end{array}$ & $p$ \\
\hline $\operatorname{Age}^{\dagger}$ & $60.96(7.95,41-85)$ & $64.47(9.32,40-94)$ & $<0.001$ \\
\hline Male sex & $242(94.9 \%)$ & $1208(96.2 \%)$ & 0.345 \\
\hline \multicolumn{4}{|l|}{ Race } \\
\hline White & $220(86.2 \%)$ & $1041(82.9 \%)$ & \\
\hline Black & $4(1.6 \%)$ & $57(4.5 \%)$ & 0.027 \\
\hline Other/Unknown & $31(12.2 \%)$ & $158(12.6 \%)$ & 0.721 \\
\hline \multicolumn{4}{|l|}{$\mathrm{BMI}^{\dagger}$} \\
\hline Underweight & $11(4.3 \%)$ & $102(8.1 \%)$ & 0.024 \\
\hline Overweight & $61(23.9 \%)$ & $320(25.5 \%)$ & 0.318 \\
\hline Obese & $96(37.6 \%)$ & $453(36.0 \%)$ & 0.640 \\
\hline \multicolumn{4}{|l|}{ Tobacco control treatments } \\
\hline Brief counseling at discharge & $245(96.1 \%)$ & $1077(85.7 \%)$ & $<0.001$ \\
\hline Smoking cessation medications during stay & $114(44.7 \%)$ & $150(11.9 \%)$ & $<0.001$ \\
\hline Smoking cessation medications dispensed in the past year & $71(27.8 \%)$ & $347(27.6 \%)$ & 0.944 \\
\hline \multicolumn{4}{|l|}{ Psychosocial Markers } \\
\hline History of alcohol abuse & $58(22.7 \%)$ & $290(23.1 \%)$ & 0.905 \\
\hline History of drug abuse & $40(15.7 \%)$ & $163(13.0 \%)$ & 0.248 \\
\hline History of homelessness & $20(7.8 \%)$ & $91(7.2 \%)$ & 0.739 \\
\hline History of psychosis & $38(14.9 \%)$ & $284(22.6 \%)$ & 0.006 \\
\hline History of depression & $18(7.1 \%)$ & $57(4.5 \%)$ & 0.091 \\
\hline History of PTSD & $45(17.6 \%)$ & $210(16.7 \%)$ & 0.719 \\
\hline \multicolumn{4}{|l|}{ Comorbidities } \\
\hline History of CHF & $34(13.3 \%)$ & $278(22.1 \%)$ & 0.002 \\
\hline History of hypertension & $120(47.1 \%)$ & $751(59.8)$ & $<0.001$ \\
\hline History of sleep apnea & $11(4.3)$ & $61(4.9)$ & 0.711 \\
\hline History of stroke & $11(4.3)$ & $109(8.7)$ & 0.019 \\
\hline History of CAD & $51(20.0)$ & 358 (28.5) & 0.005 \\
\hline History of lung cancer & $7(2.7)$ & $49(3.9)$ & 0.373 \\
\hline History of metastatic cancer & $2(0.8 \%)$ & $29(2.3 \%)$ & 0.117 \\
\hline \multirow{2}{*}{\multicolumn{4}{|c|}{ Markers of COPD severity }} \\
\hline & & & \\
\hline Count of inhaled medications & & & 0.110 \\
\hline $0-1$ & $166(65.1)$ & $904(72.0)$ & \\
\hline $2-3$ & $89(34.9)$ & $352(28.0)$ & \\
\hline Corticosteroids in the past year & $82(32.2 \%)$ & $485(38.6 \%)$ & 0.052 \\
\hline Mechanical ventilation during stay & $9(3.5)$ & $47(3.7)$ & 0.870 \\
\hline NIPPV during stay & $30(11.8)$ & $132(10.5)$ & 0.555 \\
\hline
\end{tabular}

*Unless indicated, numbers presented are $n$, \%. Bold typeface indicates statistical significance

$\uparrow$ numbers presented are mean, standard deviation and range

$\$ 2.9 \%$ missing

BMI Body mass index, PTSD post-traumatic stress disorder, CHF congestive heart failure, CAD coronary artery disease, COPD chronic obstructive pulmonary disease, NIPPV non-invasive positive pressure ventilation 


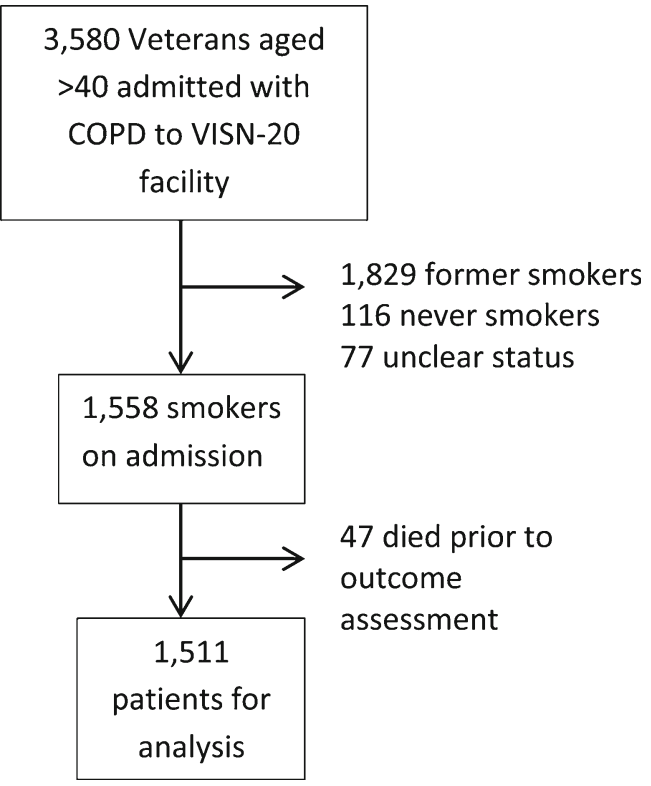

Figure 1. Results of cohort selection among patients admitted to the Veterans Affairs Veterans' Integrated Service Network 20 for exacerbation of COPD

cessation medications. Less than half of subjects treated with medications as an inpatient were dispensed medication after discharge $(44.7 \%, n=114)$. Following discharge, of those patients who received a medication $(n=255)$, the majority $(74.1 \%, n=189)$ were dispensed monotherapy with the nicotine patch, with the remainder receiving other therapies including combination therapy (Figs. 1 and 2).

\section{Disparities in Treatment with Tobacco Cessation Medications}

In adjusted analyses, several demographic characteristics were associated with decreased odds of receiving medications. For each year older, the odds of receiving medications
Table 2. Predictors of Treatment with Tobacco Cessation Medications Within 48 Hours of Discharge for Exacerbation of COPD $^{*} n_{\text {tot }}=1511$

\begin{tabular}{|c|c|c|c|}
\hline & \multicolumn{2}{|c|}{$\begin{array}{l}\text { Adjusted } \\
\text { analyses }\end{array}$} & \multirow[t]{2}{*}{$p$} \\
\hline & OR & $(95 \% \mathrm{CI})$ & \\
\hline \multicolumn{4}{|l|}{ Patient Characteristics: } \\
\hline Male sex & 1.17 & $0.43-3.27$ & 0.771 \\
\hline Race & & & \\
\hline White & 1.00 & referent & \\
\hline Black & 0.34 & $0.12-0.97$ & 0.043 \\
\hline Other/Unknown & 0.90 & $0.61-1.33$ & 0.593 \\
\hline Age (per year older) & 0.96 & $0.95-0.98$ & $<0.001$ \\
\hline Charlson Score (per point higher) & 0.89 & $0.82-0.96$ & 0.003 \\
\hline Past year history of: & & & \\
\hline Hypertension & 0.75 & $0.62-0.90$ & 0.002 \\
\hline Psychosis & 0.40 & $0.31-0.52$ & $<0.001$ \\
\hline Oral or intravenous corticosteroids & 0.80 & $0.70-0.90$ & $<0.001$ \\
\hline Drug abuse & 1.30 & $0.94-1.79$ & 0.108 \\
\hline Cerebrovascular accident & 0.71 & $0.50-1.02$ & 0.061 \\
\hline \multicolumn{4}{|l|}{ Inpatient tobacco control processes: } \\
\hline $\begin{array}{l}\text { Cessation medications while } \\
\text { inpatient }\end{array}$ & 5.95 & $\begin{array}{l}3.19- \\
11.10\end{array}$ & $<0.001$ \\
\hline $\begin{array}{l}\text { Nurse-based counseling at } \\
\text { discharge }\end{array}$ & 3.08 & $2.02-4.68$ & $<0.001$ \\
\hline
\end{tabular}

* Bold typeface indicates significance. Analyses performed using logistic regression model building with pre-specified inclusion of demographic variables age, sex, and race

at discharge declined by approximately $4 \%$ (OR 0.96, $95 \%$ CI 0.95-0.98). In comparison to white patients, black patients had significantly lower odds of receiving medications at discharge (OR 0.34, $95 \%$ CI 0.12-0.97). Patients with a history of psychosis were less likely to be dispensed medications (OR 0.40, $95 \%$ CI 0.31-0.52). Among patients with higher comorbidity index, each additional point was associated with lower odds of treatment (OR 0.89, $95 \%$ CI 0.82-0.96). Finally, several comorbidities and markers of COPD severity were associated with decreased odds of receiving medications, including hypertension, and treatment with steroids in the past year (Table 2).

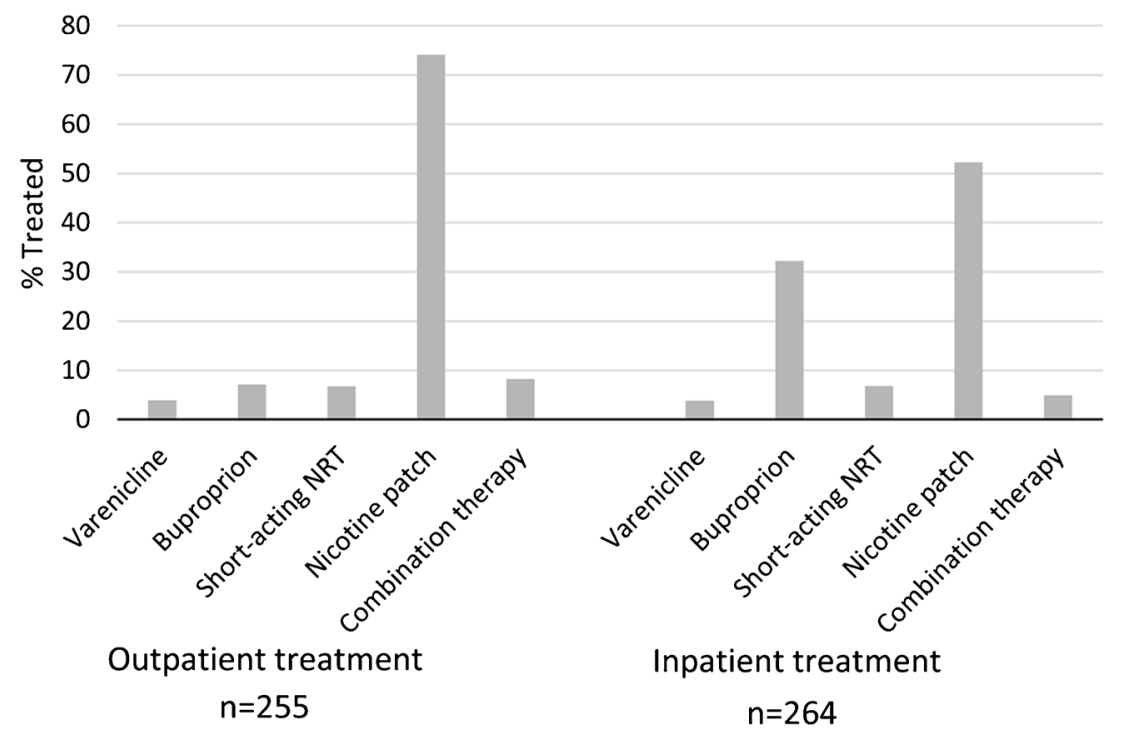

Figure 2. Among Veterans hospitalized for exacerbation of COPD who were treated with medications, choice of tobacco cessation medications provided 1) within 48 hours of discharge and 2) during hospitalization. NRT = nicotine replacement therapy 


\section{Association of Tobacco Control Processes with Receipt of Medications}

Patients who underwent documented, nurse-provided counseling prior to discharge were significantly more likely to be dispensed tobacco cessation medications after discharge in comparison to those who were not (OR 3.08, $95 \%$ CI 2.02 4.68). The strongest predictor of receipt of medications after discharge was treatment with tobacco cessation medications as an inpatient (OR 5.95, 95 \% CI 3.19-11.11). (Table 2)

\section{Sensitivity Analyses}

Given the possibility of misclassification of patients treated with buproprion for depression as patients treated for tobacco addiction, the analysis was repeated excluding treatment with buproprion from the outcome. We found a similar relationship with inpatient treatment and post-discharge treatment (OR $6.17,95 \%$ CI 3.18-12.01), with no significant differences in the other predictors. In addition, when the model building was repeated among only those subjects with a primary discharge diagnosis of COPD $(n=1087)$, there were no significant differences in inference or magnitude of the observed effects.

\section{DISCUSSION}

In this large cohort of Veteran smokers discharged following an exacerbation of COPD, we found a low rate of treatment for smoking cessation with medications, both during the hospitalization and following discharge. Black race, older age, psychiatric disease, and medical comorbidity were associated with fewer tobacco cessation medications dispensed at discharge, while the receipt of inpatient tobacco control processes was strongly associated with more tobacco cessation medications dispensed.

The proportion of patients treated with medications for nicotine withdrawal during the inpatient hospital stay was very low, though similar to previous studies examining the use of these medications among smokers admitted to the hospital. ${ }^{19-}$ ${ }^{21}$ Most smokers with COPD have significant tobacco dependence. ${ }^{22,23}$ Untreated patients are therefore likely suffering from significant withdrawal symptoms during their stay, ${ }^{24}$ or continuing to smoke while hospitalized, ${ }^{25}$ neither of which are desirable. However, we found that patients who were treated with medications for nicotine dependence during their stay had much higher odds of being dispensed cessation medications at discharge. A similar study of hospitalized patients demonstrated an increase in the use of NRT after discharge among patients treated with NRT as inpatients. ${ }^{26}$ Collectively, these data suggest that initiating treatment while in hospital may be an important intervention.

Using these medications while in the hospital may reassure patients that these cessation aids are safe and effective for them individually, and give them personal experience to draw upon when leaving the hospital. We also found a higher odds of treatment among patients who received nurse-delivered predischarge counseling. Although there is concern about the effectiveness of this low-intensity counseling intervention alone to produce sustained quit rates, ${ }^{27,28}$ an associated increase in the use of pharmacotherapy for tobacco cessation may prove to be effective, and is supported by our results. Pairing this nurse-based intervention with other processes that facilitate treatment, such as changes to electronic discharge order sets, may further improve the use of medications.

It is unclear what barriers exist to providing treatment to these patients, whether at the patient, provider, or system level. In terms of the use of the " 5 A's" of tobacco cessation, ${ }^{29}$ all of our subjects were identified as smokers in the medical record (Ask), and the majority underwent documented brief predischarge counseling (Advise), but few were ultimately provided with tobacco cessation medications (Assist). In general, the majority of hospitalized smokers indicate an interest in quitting, ${ }^{30}$ which is at odds with the low level of treatment.

Several disparities in treatment that have been identified in the outpatient setting appear to persist at discharge from an inpatient setting. Black outpatients less frequently receive advice to quit, and subsequently receive medications less often than white patients. ${ }^{31,32}$ A prior study of patients admitted for myocardial infarction indicated that black patients are less likely to be advised to quit smoking, ${ }^{7}$ suggesting that this disparity in treatment exists in other inpatient settings. Although the number of black subjects in our study was low, the difference in the odds of treatment is striking. Other studies indicate that, despite being at high risk for tobacco-related conditions, older patients and those with worse health status are counseled and treated less frequently, ${ }^{33-35}$ though this varies somewhat by setting. It is possible that our findings are explained by that patients with COPD may lack the motivation to make a quit attempt or prefer to quit without medications, and that these characteristics vary by age and race. However, it is possible that these findings represent bias on the part of physicians. ${ }^{36}$

The reasons that underlie the variations in the use of medications for tobacco cessation for patients who are older with more comorbidities are unclear, but may reflect safety concerns and fatalism on the part of patients and physicians. Though multiple studies have indicated that these therapies are safe to use in patients with a variety of medical conditions, ${ }^{37,38}$ many patients have erroneous beliefs that nicotine replacement is dangerous or ineffective, ${ }^{39,40}$ and these beliefs may be more common among patients who are older and sicker. Physicians and patients who are faced with continued tobacco use by a patient of advanced age or with significant medical comorbidity may feel fatalistic about the success and benefits of quitting. ${ }^{41}$ This may in turn contribute to lower rates of treatment. Finally, patients who are older and sicker have more medical conditions competing for physician time and attention, which can limit the time available for discussion of tobacco use. The use of dedicated counselors, rather than dependence on physicians, may help alleviate this difficulty. 
The lower observed odds of treatment among patients with a history of psychosis was unsurprising. Psychiatric patients have extremely high rates of tobacco use compared to the general population, ${ }^{42}$ and suffer from well-documented difficulties in trying to quit. ${ }^{43}$ Data indicate that psychiatric patients state an interest in quitting that is similar to smokers overall, but are unlikely to receive medications to treat nicotine addiction. ${ }^{44}$ This may stem from providers' perceptions that treating these patients is futile or may worsen their psychiatric symptoms. ${ }^{45}$ Therefore, this group may need tailored interventions to ensure that they are adequately treated for tobacco abuse.

This study has several limitations related to the observational nature of the data. We know whether patients received treatment during or after admission, but we do not know the reasons behind lack of treatment. We do not have information on patient motivation, level of addiction, or whether medications were offered by hospital staff and refused. Patients were identified administratively, and data was drawn from administrative records and chart notes that were not documented for research purposes. Measurement of tobacco status was selfreported as part of routine care, though prior data indicates this form of assessing smoking status in from healthcare interactions is generally valid. ${ }^{46,47}$ Moreover, we examined selfreported smokers, and social desirability more often leads smokers to report being non-smokers. If patients purchased nicotine replacement or obtained prescription medications outside of the VA system, this would not have been captured. We feel this is likely to be rare among patients admitted to a VA facility due to financial incentives, ${ }^{48}$ and we have excellent capture of medications provided within the VA system. Finally, although representative of the Veterans who seek care in the VISN-20, there were very few women and Hispanic patients included, which limits the ability to generalize to these groups. Women are commonly found to suffer from disparities in tobacco treatment, ${ }^{34,49}$ which would have been poorly captured in our study.

This study has several strengths. We were able to assess the provision of tobacco cessation treatment within an entire VISN, comprised of varied hospital settings including academic and rural centers. We included all smokers with a COPD exacerbation, regardless of motivation to quit. All data collection occurred after the initiation of the first JCAHO quality measures surrounding tobacco treatment in 2004, when tobacco treatment among inpatients became a priority. Finally, we have excellent completeness of pharmacy records during the hospital stay and at the time of discharge.

In summary, few patients were dispensed medications to treat tobacco addiction among this cohort of Veteran smokers discharged following exacerbation of COPD. Although our study was performed in a VA setting, similar disparities in treatment have been found in a wide variety of settings, including nationally representative samples, ${ }^{9}$ ambulatory settings, ${ }^{10}$ and other groups of hospitalized patients, ${ }^{6,7}$ suggesting that these results may be generalizable to other hospital practice settings. In addition, while our subjects had high rates of mental illness, this is also typical of the population of smokers ${ }^{50,51}$ and the population of patients with COPD. ${ }^{52,53} \mathrm{We}$ found a strong association between inpatient tobacco control processes and the provision of pharmacotherapy for smoking cessation after discharge. This suggests that mechanisms to promote guideline adherent tobacco control policies during hospitalization can have beneficial effects on use of these medications after discharge. At a system level, our data suggest that hospitals may implement novel treatment approaches, such "opt out" strategies for treatment of tobacco cessation treatment while hospitalized. Other interventions may focus on the use of integrated electronic order sets for tobacco control, dedicated inpatient tobacco counselors, and education initiatives for patients and staff. Further research is needed to help improve the delivery of tobacco cessation medications and counseling to inpatients, and to alleviate the observed disparities in treatment.

Acknowledgements: This material is based upon work supported by the Department of Veterans Affairs, Health Services Research and Development (HSR\&D), who provided access to data, office space, and programming and data management. The views expressed in this article are those of the authors and do not necessarily reflect the position or policy of the Department of Veterans Affairs. We would like to acknowledge Mr. Robert Plumley, who performed the data extraction and natural language processing necessary to complete this project. Dr. Au is an unpaid research consultant for Analysis Group. Dr. Melzer is supported by an institutional F-32 (HL007287-36) through the University of Washington Department of Pulmonary and Critical Care. Dr. Feemster is supported by an NIH NHLBI K23 Mentored Career Development Award (HL111116). Partial support of this project was provided by Gilead Sciences with research funding to the Seattle Institute for Biomedical and Clinical Research. Additional support was received through the VA Health Services Research and Development (HSR\&D).

Corresponding Author: Anne C. Melzer, MD; Center of Innovation for Veteran-Centered and Value-Driven CareVA Puget Sound Health Care System, 1660 S. Columbian Way, Mailstop S-152, Seattle, WA 98108 , USA (e-mail: acmelzer@uw.edu).

\section{Compliance with Ethical Standards:}

Conflicts of Interest: The authors declare that they do not have a conflict of interest.

\section{REFERENCES}

1. Murphy SL, Xu JU, Kochanek KD. Deaths: Final data for 2010. Hyattsville: National Center for Health Statistics; 2013.

2. Anthonisen NR, Connett JE, Kiley JP, et al. Effects of smoking intervention and the use of an inhaled anticholinergic bronchodilator on the rate of decline of FEV1: The lung health study. JAMA. 1994;272:1497505.

3. Garcia-Aymerich J, Barreiro E, Farrero E, Marrades R, Morera J, Anto J. Patients hospitalized for COPD have a high prevalence of modifiable risk factors for exacerbation (EFRAM study). Eur Respir J. 2000;16:1037-42.

4. Yip NH, Yuen G, Lazar EJ, et al. Analysis of Hospitalizations for COPD Exacerbation: Opportunities for Improving Care. COPD: J Chron Obstructive Pulm Dis. 2010;7:85-92.

5. Emmons KM, Goldstein MG. Smokers who are hospitalized: a window of opportunity for cessation interventions. Prev Med. 1992;21:262-9.

6. Freund M, Campbell E, Paul C, et al. Smoking care provision in hospitals: a review of prevalence. Nicotine Tob Res Off $\mathrm{J}$ Soc Res Nicotine Tob. 2008; 10:757-74. 
7. Sonel AF, Good CB, Mulgund J, et al. Racial variations in treatment and outcomes of black and white patients with high-risk non-ST-elevation acute coronary syndromes: insights from CRUSADE (Can Rapid Risk Stratification of Unstable Angina Patients Suppress Adverse Outcomes With Early Implementation of the ACC/AHA Guidelines?). Circulation. 2005; 111:1225-32.

8. Moolchan ET, Fagan P, Fernander AF, et al. Addressing tobacco-related health disparities. Addict (Abingdon, Engl) 2007;102 Suppl 2:30-42.

9. Cokkinides VE, Halpern MT, Barbeau EM, Ward E, Thun MJ. Racial and Ethnic Disparities in Smoking-Cessation Interventions: Analysis of the 2005 National Health Interview Survey. Am J Prev Med. 2008;34:404-12.

10. Fu SS, Sherman SE, Yano EM, van Ryn M, Lanto AB, Joseph AM. Ethnic disparities in the use of nicotine replacement therapy for smoking cessation in an equal access health care system. Am J Health Promot. 2005;20: 108-16.

11. Eisner MD, Blanc PD, Omachi TA, et al. Socioeconomic status, race and COPD health outcomes. J Epidemiol Commun Health.2009.

12. Collins BF, Ramenofsky D, Au DH, Ma J, Uman JE, Feemster LC. The association of weight with the detection of airflow obstruction and inhaled treatment among patients with a clinical diagnosis of COPD. Chest 2014.

13. Herrin MA, Feemster LC, Crothers K, Uman JE, Bryson CL, Au DH. Combination antihypertensive therapy among patients with COPD. Chest. 2013;143:1312-20.

14. Melzer AC, Feemster LM, Uman JE, Ramenofsky DH, Au DH. Missing potential opportunities to reduce repeat COPD exacerbations. J Gen Intern Med. 2013;28:652-9.

15. McGinnis KA, Brandt CA, Skanderson M, et al. Validating smoking data from the Veteran's Affairs Health Factors dataset, an electronic data source. Nicotine Tob Res Off J Soc Res Nicotine Tob. 2011;13:1233-9.

16. Elixhauser A, Steiner C, Harris DR, Coffey RM. Comorbidity measures for use with administrative data. Med Care. 1998;36:8-27.

17. Deyo RA, Cherkin DC, Ciol MA. Adapting a clinical comorbidity index for use with ICD-9-CM administrative databases. J Clin Epidemiol. 1992;45:613-9.

18. Hosmer JDW, Lemeshow S, Sturdivant RX. Model-Building Strategies and Methods for Logistic Regression. Applied Logistic Regression: John Wiley \& Sons, Inc.; 2013:89-151

19. Rigotti NA, Clair C, Munafo MR, Stead LF. Interventions for smoking cessation in hospitalised patients. Cochrane Database Syst Rev 2012;16.

20. Emmons KM, Goldstein MG, Roberts M, et al. The use of nicotine replacement therapy during hospitalization. Ann Behav Med: a Publ Soc Behav Med. 2000;22:325-9.

21. Faseru B, Turner M, Casey G, et al. Evaluation of a hospital-based tobacco treatment service: outcomes and lessons learned. J Hosp Med: an Off Publ Soci Hosp Med. 2011;6:211-8.

22. Jiménez-Ruiz CA, Masa F, Miravitlles M, et al. Smoking characteristics*: Differences in attitudes and dependence between healthy smokers and smokers with COPD. CHEST J. 2001;119:1365-70.

23. Vozoris NT, Stanbrook MB. Smoking prevalence, behaviours, and cessation among individuals with COPD or asthma. Respir Med. 2011;105:47784.

24. Morrell HER, Cohen LM, al'Absi M. Physiological and psychological symptoms and predictors in early nicotine withdrawal. Pharmacol Biochem Behav. 2008;89:272-8.

25. Rigotti NA, Arnsten JH, McKool KM, Wood-Reid KM, Pasternak RC, Singer DE. Smoking by Patients in a Smoke-Free Hospital: Prevalence. Predictors, and Implications. Prev Med. 2000;31:159-66.

26. Regan S, Reyen M, Richards AE, Lockhart AC, Liebman AK, Rigotti NA. Nicotine Replacement Therapy Use at Home After Use During a Hospitalization. Nicotine Tob Res. 2012;14:885-9.

27. Stevens VJ, Glasgow RE, Hollis JF, Mount K. Implementation and Effectiveness of a Brief Smoking-Cessation Intervention for Hospital Patients. Med Care. 2000;38:451-9.

28. Reeves GR, Wang TY, Reid KJ, et al. Dissociation between hospital performance of the smoking cessation counseling quality metric and cessation outcomes after myocardial infarction. Arch Intern Med. 2008; 168:2111-7.

29. Fiore MC, Jaen CR, Baker TB, Baily WC, Benowitz NL, Curry SJ, et al. Treating Tobacco Use and Dependence: 2008 Update. U.S. Department of Health and Human Services. Public Health Service; Rockville, MD: 2008.
30. Sciamanna CN, Hoch JS, Duke GC, Fogle MN, Ford DE. Comparison of Five Measures of Motivation to Quit Smoking Among a Sample of Hospitalized Smokers. J Gen Intern Med. 2000; 15:16-23.

31. Lopez-Quintero C, Crum RM, Neumark YD. Racial/Ethnic Disparities in Report of Physician-Provided Smoking Cessation Advice: Analysis of the 2000 National Health Interview Survey. Am J Public Health. 2006;96:2235-9.

32. Trinidad DR, Perez-Stable EJ, White MM, Emery SL, Messer K. A nationwide analysis of US racial/ethnic disparities in smoking behaviors, smoking cessation, and cessation-related factors. Am J Public Health. 2011;101:699-706.

33. Doescher MP, Saver BG. Physicians' advice to quit smoking. The glass remains half empty. J Fam Pract. 2000;49:543-7.

34. Steinberg MB, Akincigil A, Delnevo CD, Crystal S, Carson JL. Gender and Age Disparities for Smoking-Cessation Treatment. Am J Prev Med. 2006;30:405-12.

35. Houston TK, Scarinci IC, Person SD, Greene PG. Patient smoking cessation advice by health care providers: the role of ethnicity, socioeconomic status, and health. Am J Public Health. 2005;95: 1056-61.

36. Blair IV, Steiner JF, Fairclough DL, et al. Clinicians' implicit ethnic/ racial bias and perceptions of care among Black and Latino patients. Ann Fam Med. 2013;11:43-52.

37. McRobbie H, Hajek P. Nicotine replacement therapy in patients with cardiovascular disease: guidelines for health professionals. Addict (Abingdon, Engl). 2001;96:1547-51.

38. Joseph AM, Norman SM, Ferry LH, et al. The safety of transdermal nicotine as an aid to smoking cessation in patients with cardiac disease. $\mathrm{N}$ Engl J Med. 1996;335:1792-8.

39. Bansal MA, Cummings KM, Hyland A, Giovino GA. Stop-smoking medications: who uses them, who misuses them, and who is misinformed about them? Nicotine Tob Res Off J Soc Res Nicotine Tob. 2004;6(Suppl 3):S303-10

40. Shiffman S, Ferguson SG, Rohay J, Gitchell JG. Perceived safety and efficacy of nicotine replacement therapies among US smokers and exsmokers: relationship with use and compliance. Addict (Abingdon, Engl). 2008; 103:1371-8

41. Wilson JS, Elborn JS, Fitzsimons D. 'It's not worth stopping now': why do smokers with chronic obstructive pulmonary disease continue to smoke? A qualitative study. J Clin Nurs. 2011;20:819-27.

42. Lasser K, Boyd J, Woolhandler S, Himmelstein DU, McCormick D, Bor DH. Smoking and mental illness: A population-based prevalence study. JAMA. 2000;284:2606-10.

43. Kupiainen H, Kinnula VL, Lindqvist A, et al. Successful smoking cessation in COPD: association with comorbidities and mortality. Pulm Med. 2012;2012:725024.

44. Prochaska JJ, Fletcher L, Hall SE, Hall SM. Return to smoking following a smoke-free psychiatric hospitalization. Am J Addict. 2006;15:15-22.

45. Prochaska JJ. Smoking and mental illness-breaking the link. N Engl J Med. 2011;365:196-8.

46. Studts JL, Ghate SR, Gill JL, et al. Validity of Self-reported Smoking Status among Participants in a Lung Cancer Screening Trial. Cancer Epidemiol Biomark Prev. 2006;15:1825-8.

47. Yeager DS, Krosnick JA. The validity of self-reported nicotine product use in the 2001-2008 National Health and Nutrition Examination Survey. Med Care. 2010;48:1128-32.

48. Shen YH, A. Zhang, S. Kazis, L. VHA Enrollees' Health Care Coverage and Use of Care. Med Care Res Rev 2003;60.

49. Sherman SE, Fu SS, Joseph AM, Lanto AB, Yano EM. Gender differences in smoking cessation services received among veterans. Womens Health Issues. 2005; 15:126-33.

50. Lawrence D, Mitrou F, Zubrick SR. Smoking and mental illness: results from population surveys in Australia and the United States. BMC Public Health. 2009;9:285.

51. Control CfD, Prevention. Vital signs: current cigarette smoking among adults aged $\geq 18$ years with mental illness-United States, 2009-2011. MMWR Morbidity and mortality weekly report 2013;62:81.

52. Himelhoch S, Lehman A, Kreyenbuhl J, Daumit G, Brown C, Dixon L. Prevalence of chronic obstructive pulmonary disease among those with serious mental illness. Am J Psychiatry. 2004;161:2317-9.

53. Sokal J, Messias E, Dickerson FB, et al. Comorbidity of medical illnesses among adults with serious mental illness who are receiving community psychiatric services. J Nerv Ment Dis. 2004;192:421-7. 